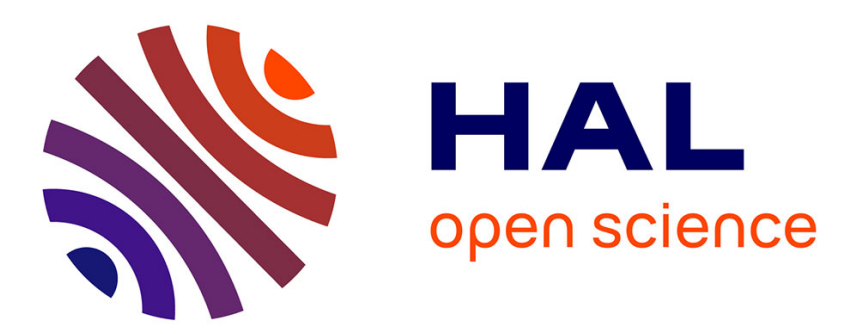

\title{
Semi-Supervised Hyperspectral Image Segmentation Using Regionalized Stochastic Watershed
}

\author{
Jesus Angulo, Santiago Velasco-Forero
}

\section{To cite this version:}

Jesus Angulo, Santiago Velasco-Forero. Semi-Supervised Hyperspectral Image Segmentation Using Regionalized Stochastic Watershed. Algorithms and Technologies for Multispectral, Hyperspectral, and Ultraspectral Imagery XVI, May 2010, Orlando, United States. 12 p., 10.1117/12.850187 . hal00834482

HAL Id: hal-00834482

https://hal-mines-paristech.archives-ouvertes.fr/hal-00834482

Submitted on 15 Jun 2013

HAL is a multi-disciplinary open access archive for the deposit and dissemination of scientific research documents, whether they are published or not. The documents may come from teaching and research institutions in France or abroad, or from public or private research centers.
L'archive ouverte pluridisciplinaire $\mathbf{H A L}$, est destinée au dépôt et à la diffusion de documents scientifiques de niveau recherche, publiés ou non, émanant des établissements d'enseignement et de recherche français ou étrangers, des laboratoires publics ou privés. 


\title{
Semi-supervised hyperspectral image segmentation using regionalized stochastic watershed
}

\author{
Jesús Angulo $^{a}$ and Santiago Velasco-Forero ${ }^{a}$ \\ ${ }^{a}$ CMM-Centre de Morphologie Mathématique, Mathématiques et Systèmes, MINES ParisTech; \\ 35, rue Saint-Honoré, 77305 Fontainebleau cedex - France
}

\begin{abstract}
Stochastic watershed is a robust method to estimate the probability density function (pdf) of contours of a multi-variate image using MonteCarlo simulations of watersheds from random markers. The aim of this paper is to propose a stochastic watershed-based algorithm for segmenting hyperspectral images using a semi-supervised approach. Starting from a training dataset consisting in a selection of representative pixel vectors of each spectral class of the image, the algorithm calculate for each class a membership probability map (MPM). Then, the MPM of class $k$ is considered as a regionalized density function which is used to simulate the random markers for the MonteCarlo estimation of the pdf of contours of the corresponding class $k$. This pdf favours the spatial regions of the image spectrally close to the class $k$. After applying the same technique to each class, a series of pdf are obtained for a single image. Finally, the pdf's can be segmented hierarchically either separately for each class or after combination, as a single pdf function. In the results, besides the generic spatial-spectral segmentation of hyperspectral images, the interest of the approach is also illustrated for target segmentation.
\end{abstract}

Keywords: hyperspectral images, semi-supervised segmentation, stochastic watershed, regionalized random germs, probabilistic segmentation

\section{INTRODUCTION}

Watershed transformation is one of the most powerful tools for image segmentation. Starting from a gradient, the classical paradigm of watershed segmentation consists in determining markers for each region of interest. The markers avoid the over-segmentation (a region is associated to each minimum of the function) and moreover, the watershed is relatively robust to marker position. ${ }^{3}$ The segmentation by watershed of hyperspectral images has shown to improve the results of classification in hyperspectral images. ${ }^{15,16}$ To deals with some drawbacks of the classical deterministic watershed, the stochastic watershed ${ }^{1}(\mathrm{SW})$ was introduced to detect and to regularize the contours which are robust with respect to variations in the segmentation conditions. The initial framework was then extended to multispectral images ${ }^{11}$ and later, a specific methodology of classification-driven stochastic watershed for multivariate images such as multi/hyper-spectral images was also proposed. ${ }^{12}$ More recently, we have also considered $i^{2}$ a probabilistic multiscale framework for the computation of the probability of contours using different multiscale frameworks of stochastic watershed.

Fig. 1 depicts two standard examples of remote sensing hyperspectral images which will be used in the study. The first dataset is the hyperspectral image of the Indian Pines (200 spectral bands in the 400-2500 nm range, 145x145 pixels), obtained by the AVIRIS sensor. This is a typical example of high spectral resolution image but with a low spatial resolution, which is particularly useful, for instance, in remote sensing agricultural applications. The other dataset is an airbone image, from the ROSIS-3 optical sensor, of the University of Pavia (103 spectral bands of 340x610 pixels). This kind of high spatial resolution image can be typically considered in remote sensing geography or military applications. In the same figure are given, on the one hand, a false colour image with the ground truth pixel classification, and on the other hand, the image of contours of each region of the spectral classification. To be more precise, given a certain set of spectral classes, the pixels belonging to the same spectral class are manually labelled with the same "colour" (the background here is in white). This kind

Further author information: (Send correspondence to J.A. or to S.V.-F.)

J. Angulo: E-mail: jesus.angulo@mines-paristech.fr

S. Velasco-Forero: E-mail: santiago.velasco@mines-paristech.fr 


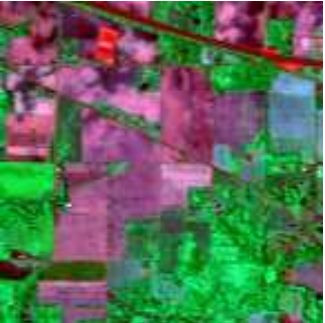

(a1)

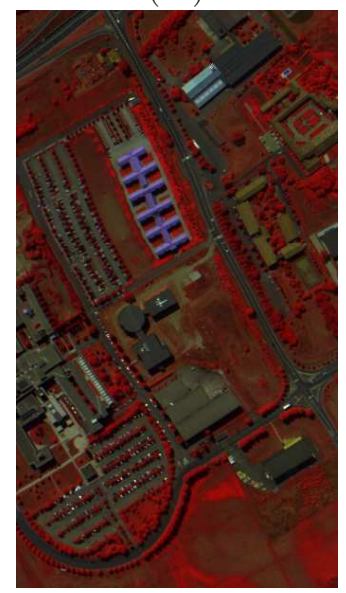

$(\mathrm{a} 2)$

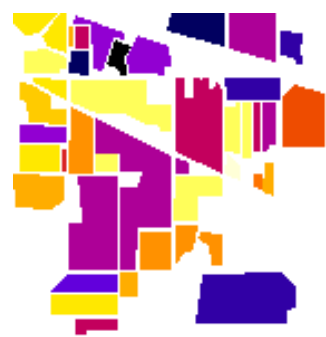

(b1)

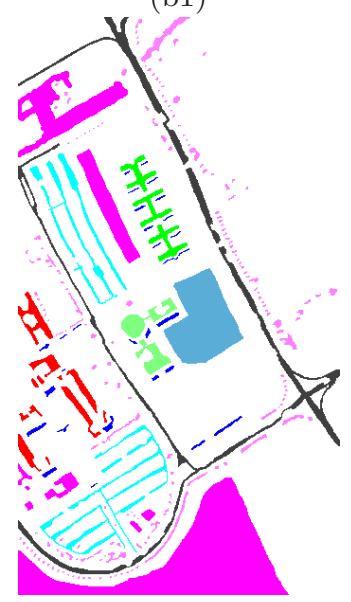

(b2)

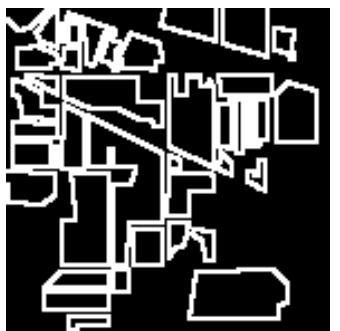

$(\mathrm{c} 1)$

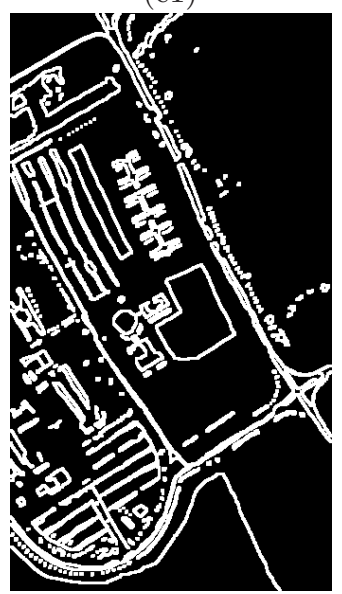

(c2)

Figure 1. Two standard examples of remote sensing hyperspectral images: (1) "Indian Pines" and (2) "Pavia". The false colour images (a) are obtained from three significant spectral bands. The spectral classes defined by the ground truth classification are given in images (b): 16 classes for "Indian Pines" and 9 classes for "Pavia" (the background class appears in white). The images (c) represent the contours of the regions associated to the ground truth classification.

of reference is very useful to evaluate the performance of pixel classification algorithms. However, as we observe from both examples, there are many spatial structures which clearly appear on the original images but which are not associated to contours of the spectral classes to be separated, e.g., regions typically associated to the "background" spectral class.

Image segmentation can be considered under two different paradigms: unsupervised and supervised. In unsupervised image segmentation, the aim is to obtain in a reliable way the contours of the most significant spatial/spectral structures. In high spatial resolution images, as the example "Pavia", there are many complex structures at various scales and the definition of a single segmentation is a challenging difficult problem. In supervised image segmentation, some prior information is introduced to help the segmentation procedure. Typically, this prior knowledge corresponds to: i) "markers" for the regions of interest, in the case of interactive segmentation; or ii) to a pixel classification in homogenous regions, which are the germs for an automatic segmentation.

Hyperspectral imaging is an active field of image analysis which is usually considered under the supervised paradigm: both the complexity of the data and the typical real-life applications require the construction of training sets of spectra which drive the algorithms of classification, feature extraction, segmentation, etc. In this paper we would like to be coherent with this paradigm, and we use the prior information from a spectral training dataset in order to produce a kind of hyperspectral segmentation using the stochastic watershed-based algorithm. In fact, our method does not include a deterministic classification step of pixels and this is reason why we consider the approach as a semi-supervised segmentation; however our technique is not related to the semi-supervised learning paradigm of classification. ${ }^{5}$

More precisely, starting from a training dataset consisting in a selection of representative pixel vectors of 
each spectral class of the image, the algorithm calculate for each class a membership probability map (MPM). Then, the MPM of class $k$ is considered as a regionalized density function which is used to simulate the random markers used in the MonteCarlo estimation of the pdf of contours of the corresponding class $k$. This pdf favours the spatial regions of the image close spectrally to the class $k$. After applying the same technique to each class, a series of pdf are obtained for a single image. Finally, the pdf's can be segmented hierarchically either separately for each class or after combination, as a single pdf function. In the results, besides the generic spatial-spectral segmentation of hyperspectral images, the interest of the approach is also illustrated for target segmentation.

\section{REMIND ON UNSUPERVISED STOCHASTIC WATERSHED FOR MULTI/HYPER-SPECTRAL IMAGES}

Hyperspectral images are multivariate discrete functions with typically several tens or hundreds of spectral bands. Let $\mathbf{f}_{\lambda}(\mathbf{x})=\left\{f_{\lambda_{j}}(\mathbf{x})\right\}_{j=1}^{L}$ be a hyperspectral image, with $\mathbf{f}_{\lambda}: E \rightarrow \mathcal{T}^{L}$, where $\mathbf{x}=(x, y)$ are the spatial coordinates of a vector pixel, such that $\mathbf{x} \in E \subset \mathbb{Z}^{2}$; the space of spectral values is $\mathcal{T}^{L} \subset \mathbb{R}^{L}$. The scalar image $f_{\lambda_{j}}(\mathbf{x})$ corresponds to the channel or band $j, j \in\{1,2, \ldots, L\}$. Let $g(\mathbf{x})$ and $m r k(\mathbf{x})$ be a gradient image and a marker image, respectively. Intuitively, the associated watershed transformation, $W S(g, m r k)$, produces a binary image with the contours of regions "marked" by the image mrk according to the "energy of contour" given by the gradient image $g .{ }^{3}$ In mathematical morphology, the gradient $\varrho(f)(\mathbf{x})$ of an image $f(\mathbf{x})$ is obtained as the pointwise difference between an unit dilation and an unit erosion, i.e.,

$$
f(\mathbf{x}) \mapsto \varrho(f)(\mathbf{x})=\delta_{B}(f)(\mathbf{x})-\varepsilon_{B}(f)(\mathbf{x}) .
$$

In addition, we consider that the values of the gradient image $\varrho(f)$ are defined in the interval $[0,1]$. The image $m r k(\mathbf{x})$ is composed of binary connected components.

The classical paradigm of watershed segmentation lays on the appropriate choice of markers, which are the seeds to initiate the flooding procedure. In the stochastic watershed approach, an opposite direction is followed, by selecting random germs for markers on the watershed segmentation. This arbitrary choice will be balanced by the use of a given number $M$ of realizations, in order to filter out non significant fluctuations. Each piece of contour may then be assigned the number of times it appears during the various simulations in order to estimate a probability density function (pdf) of contours. Large regions, separated by low contrast gradient from neighbouring regions will be sampled more frequently than smaller regions and will be selected more often. On the other hand, strong contours will often be selected by the watershed construction, as there are many possible positions of markers which will select them. So the probability of selecting a contour will offer a nice balance between strength of the contours and size of the adjacent

More precisely, given the scalar image of a spectral band, $f_{\lambda_{j}}$, the associated pdf of contours is obtained as follows. Let $\left\{m r k_{i}(\mathbf{x})\right\}_{i=1}^{M}$ be a series of $M$ realizations ( $M$ binary images) of $N$ spatially uniformly distributed random markers. Each one of these binary images of points is considered as the markers for a watershed segmentation of gradient image $\varrho\left(f_{\lambda_{j}}\right)$ such that the obtained image

$$
W S\left(\varrho\left(f_{\lambda_{j}}\right), m r k_{i}\right)(\mathbf{x})= \begin{cases}1 & \text { if } \mathbf{x} \in \mathrm{WS} \text { contours } \\ 0 & \text { Otherwise }\end{cases}
$$

Consequently, a series of $M$ segmentations is obtained, i.e., $\left\{W S\left(\varrho\left(f_{\lambda_{j}}\right), m r k_{i}\right)\right\}_{i=1}^{M}$. Note that in each realization the number of points determines the number of regions obtained (i.e., essential property of watershed transformation). Then, the probability density function of contours is computed by the kernel density estimation method, or Parzen window method, as follows:

$$
p d f_{\lambda_{j}}(\mathbf{x})=\frac{1}{M} \sum_{i=1}^{M} W S\left(\varrho\left(f_{\lambda_{j}}\right), m r k_{i}\right)(\mathbf{x}) * K\left(\mathbf{x} ; \sigma_{\text {spatial }}\right) .
$$

Typically, the kernel $K\left(\mathbf{x} ; \sigma_{\text {spatial }}\right)$ is a spatial Gaussian function of width $\sigma_{\text {spatial }}$, i.e.,

$$
K\left(\mathbf{x} ; \sigma_{\text {spatial }}\right)=\frac{1}{2 \pi \sigma_{\text {spatial }}^{2}} \exp \left(\frac{-\|\mathbf{x}\|^{2}}{2 \sigma_{\text {spatial }}^{2}}\right)
$$


which determines the smoothing effect. We took typically for our experiments $\sigma_{\text {spatial }}=5$. In some cases, the Gaussian kernel is important to obtain a regularized pdf of contours, where contours spatially close (e.g., in textured regions or associated to small regions) are added together.

In the case of multivariate images, such as hyperspectral images, a marginal pdf is built for each channel, according to the previous schema. Hence, the marginal probability density function of hyperspectral image $\mathbf{f}_{\lambda}(\mathbf{x})$ is computed as

$$
p d f(\mathbf{x})=\frac{1}{L} \sum_{j=1}^{L} p d f_{\lambda_{j}}(\mathbf{x}) * K\left(\lambda ; \sigma_{\text {spectral }}, \omega_{j}\right),
$$

where the kernel $K\left(\lambda ; \sigma_{\text {spectral }}, \omega_{j}\right)$ is the product of a spectral Gaussian function of width $\sigma_{\text {spectral }}$ and a scalar weight $\omega_{j} \geq 0$ for each spectral band $j$. The rationale that justified this kernel is the fact that contiguous spectral bands are similar and consequently, their marginal pdfs can be smoothed together. The set of values $\left\{\omega_{j}\right\}_{j=1}^{L}$ can be used for instance to weight the pdf according to the entropy of the band, or to the noise of the band. For simplicity, we took $\omega_{j}=1, \forall j$ and $\sigma_{\text {spectral }}=3$.

We notice that $p d f(\mathbf{x})$ gives the edge strength which can be interpreted as the probability of pixel $\mathbf{x}$ to belong to the segmentation contours, i.e., $p d f(\mathbf{x}) \equiv \operatorname{Pr}(\mathbf{x} \in$ segmentation $)$.

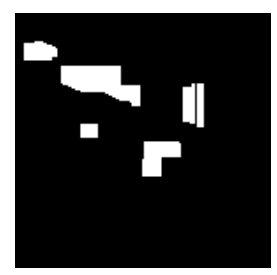

(a)

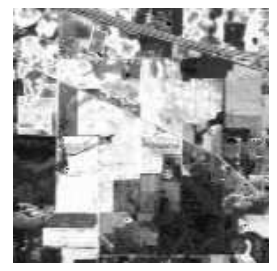

(b)

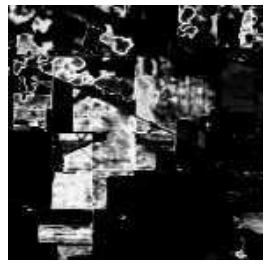

(c)

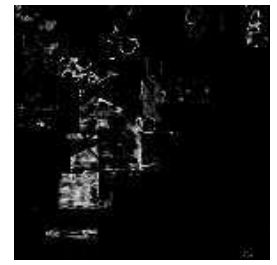

(d)

Figure 2. Influence of parameter $\sigma_{M P M}$ on the computation of MPM using Euclidean distance, $\pi_{k}^{M}(\mathbf{x})$, for spectral class 2 of "Indian Pines": (a) ground truth of the class; (b) $\sigma_{M P M}=1$, (c) $\sigma_{M P M}=0.1$, (d) $\sigma_{M P M}=0.01$.

\section{MODELLING MEMBERSHIP PROBABILITY MAP (MPM) OF SPECTRAL CLASSES}

Let us suppose that the spectral space of image $\mathbf{f}_{\lambda}(\mathbf{x})$ is composed of $K$ spectral classes: $\left\{C_{1}, C_{2}, \cdots, C_{K}\right\}$. In addition, using a training set, each spectral class $C_{k}$ is represented by a set of $n_{k}$ discrete spectra, i.e., $C_{k}=\left\{\mathbf{s}_{1, k}, \mathbf{s}_{2, k}, \cdots, \mathbf{s}_{n_{k}, k}\right\}$, such that $\mathbf{s}_{i, k} \in \mathcal{T}^{L}$.

Using a standard assumption on hyperspectral imaging, like it is considered for instance in target detection, ${ }^{8,9}$ let us suppose that the points of each spectral classes follow a normal distribution in the space $\mathcal{T}^{L} \subset \mathbf{R}^{L}$, i.e., $C_{k} \equiv \mathcal{N}\left(\mu_{k}, \Sigma_{k}\right)$ where $\mu_{k}$ is the mean spectrum and $\Sigma_{k}$ the covariance matrix of class $C_{k}$. Therefore, using Bayesian terminology, the Gaussian conditional density per class is given by

$$
\operatorname{Pr}\left(\mathbf{s}_{i} \mid C_{k}\right)=\frac{1}{(2 \pi)^{\frac{L}{2}}\left|\Sigma_{k}\right|^{\frac{L}{2}}} e^{-\frac{1}{2}\left(\mathbf{s}_{i}-\mu_{k}\right)^{T} \Sigma_{k}^{-1}\left(\mathbf{s}_{i}-\mu_{k}\right)}
$$

According to the Bayes Decision Rule, the maximum a posteriori discriminant function becomes

$$
g_{k}\left(\mathbf{s}_{i}\right)=\operatorname{Pr}\left(C_{k} \mid \mathbf{s}_{i}\right)=\frac{\operatorname{Pr}\left(\mathbf{s}_{i} \mid C_{k}\right) \operatorname{Pr}\left(C_{k}\right)}{\operatorname{Pr}\left(\mathbf{s}_{i}\right)}=\frac{1}{(2 \pi)^{\frac{L}{2}}\left|\Sigma_{k}\right|^{\frac{L}{2}}} e^{-\frac{1}{2}\left(\mathbf{s}_{i}-\mu_{k}\right)^{T} \Sigma_{k}^{-1}\left(\mathbf{s}_{i}-\mu_{k}\right)} \frac{\operatorname{Pr}\left(C_{k}\right)}{\operatorname{Pr}\left(\mathbf{s}_{i}\right)}
$$

and now, eliminating constant terms and taking natural logs (the logarithm is monotonically increasing), the classical quadratic discriminant function is obtained

$$
g_{k}\left(\mathbf{s}_{i}\right)=-\frac{1}{2}\left[\left(\mathbf{s}_{i}-\mu_{k}\right)^{T} \Sigma_{k}^{-1}\left(\mathbf{s}_{i}-\mu_{k}\right)\right]-\frac{1}{2} \log \left(\left|\Sigma_{k}\right|\right)+\log \left(\operatorname{Pr}\left(C_{k}\right)\right) .
$$


For convenience we assume also equiprobable priors so we can drop the term $\log \left(\operatorname{Pr}\left(C_{k}\right)\right)$ (if the relative "size" of each class in the image is known, the value of this term can be consequently calculated); it is also current to assume that $\left|\Sigma_{n}\right|=\left|\Sigma_{m}\right|$ for any pair of classes $n$ and $m$. Under these assumptions, the classical Mahalanobis distance classifier is obtained

$$
g_{k}\left(\mathbf{s}_{i}\right)=-\frac{1}{2}\left[\left(\mathbf{s}_{i}-\mu_{k}\right)^{T} \Sigma^{-1}\left(\mathbf{s}_{i}-\mu_{k}\right)\right] .
$$

Another example of "metric" used widely in hyperspectral data processing for classification, detection, identification, etc. is the Spectral Angle Mapper (SAM) algorithm. ${ }^{19}$ More precisely, the SAM calculates the angle between any pixel vector and a reference spectrum. It is well known that the angle (closely related also to the notion of correlation coefficient), as a normalized inner product, is an intensity independent measure: this is important for instance is the reference spectrum, as an average of representative spectra $\mu_{k}$ of class $C_{k}$, is obtained from an image or database different from the current image. Hence, the SAM classifier is given by

$$
h_{k}\left(\mathbf{s}_{i}\right)=-S A M\left(\mathbf{s}_{i}, \mu_{k}\right)=-\arccos \left(\frac{\left\langle\mathbf{s}_{i}, \mu_{k}\right\rangle}{\left\|\mathbf{s}_{i}\right\| \cdot\left\|\mu_{k}\right\|}\right)
$$

where $\langle\cdot, \cdot\rangle$ is the scalar product of two vectors and $\|\cdot\|$ is the norm of the vector.

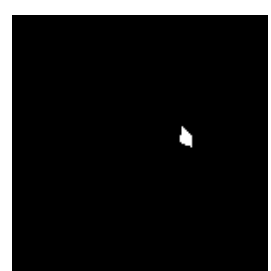

(a1)

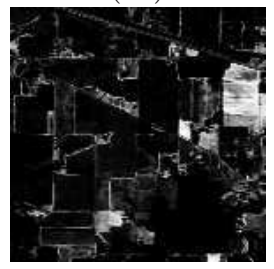

(b1)

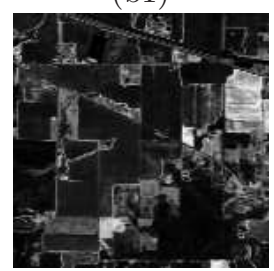

(c1)

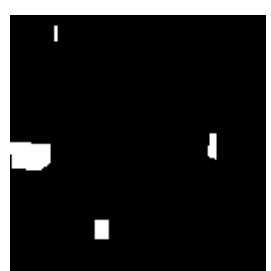

(a2)

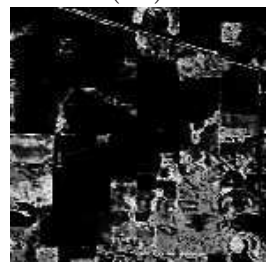

(b2)

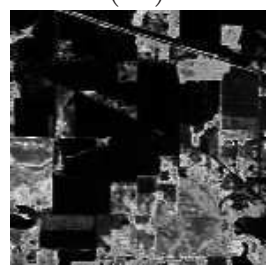

(c2)

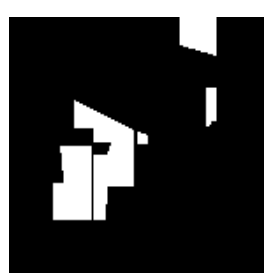

(a3)

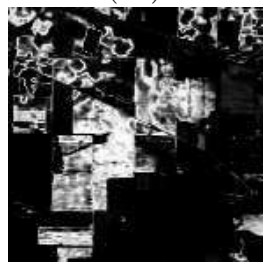

(b3)

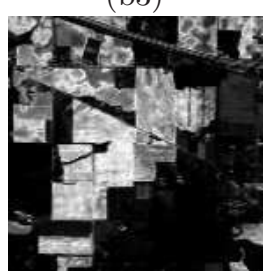

(c3)

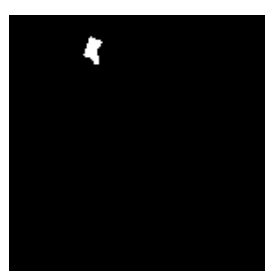

$(\mathrm{a} 4)$

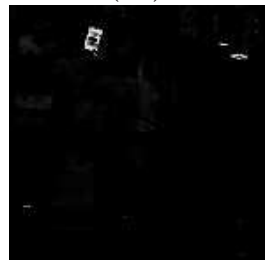

(b4)

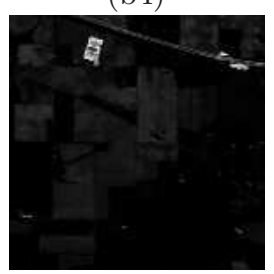

$(\mathrm{c} 4)$

Figure 3. Comparison of MPM using Euclidean distance vs. Spectral Angle Mapper, for four spectral classes of "Indian Pines" (first column corresponds to class 1, second to class 5, third to class 11 and fourth to class 16): (a) ground truth of the class; (b) $\pi_{k}^{M}(\mathbf{x})$ with $\sigma_{M P M}=0.1 ;(\mathrm{c}) \pi_{k}^{A}(\mathbf{x})$ with $\sigma_{M P M}=0.05$.

Let us come back to our objective of probabilistic segmentation. Being inspired by these classical hyperspectral classifiers, we can now calculate for each class a scalar image $\pi_{k}^{M}(\mathbf{x}): E \rightarrow \mathbb{R}_{+}$, that we name membership probability map (MPM) of the class $C_{k}$, which is defined by

$$
\pi_{k}^{M}(\mathbf{x})=\frac{\exp \left(\frac{-\frac{1}{2}\left[\left(\mathbf{f}_{\lambda}(\mathbf{x})-\mu_{k}\right)^{T} \Sigma_{k}^{-1}\left(\mathbf{f}_{\lambda}(\mathbf{x})-\mu_{k}\right)\right]}{\sigma_{M P M}}\right)}{\sum_{\mathbf{y} \in E} \exp \left(\frac{-\frac{1}{2}\left[\left(\mathbf{f}_{\lambda}(\mathbf{y})-\mu_{k}\right)^{T} \Sigma_{k}^{-1}\left(\mathbf{f}_{\lambda}(\mathbf{y})-\mu_{k}\right)\right]}{\sigma_{M P M}}\right)} .
$$


This probability density function is proportional to a Gaussian-kernel version of the Mahalanobis distance computed for each pixel of the image. A high value of $\pi_{k}(\mathbf{x})$ implies that the image value spectrum $\mathbf{f}_{\lambda}(\mathbf{x})$ has a high probability to belongs to the class $C_{k}$.

If the number of spectral samples available for each class $C_{k}$ is small (with respect to the spectral dimension), the computation of the inverse of covariance matrix $\Sigma_{k}^{-1}$ makes no sense and it may introduce important errors in the distance. A first approximation consist in considering only the band variances (i.e., $\Sigma_{k}$ is a diagonal matrix). But, in the experiments of this paper we use only 10 spectral samples as training set for each spectral class and consequently the most appropriate is to consider that $\Sigma_{k}$ is the identity matrix: in summary, $\pi_{k}^{M}(\mathbf{x})$ is based on the Euclidean distance. The parameter $\sigma_{M P M}$ allows to introduce a scaling regularization of the distance values. In Fig. 2 is depicted an example, for spectral class 2 of "Indian Pines", which illustrates the influence of parameter $\sigma_{M P M}$ on the computation of MPM using Euclidean distance, $\pi_{k}^{M}(\mathbf{x})$. The results are similar for other classes, and empirically we found that $\sigma_{M P M}=0.1$ produces a good trade-off. We can also notice that this simple metric is not very specific for similar spectral classes: in the current example, several spectral regions of "corn vegetation" produces close values of $\pi_{k}^{M}(\mathbf{x})$.

Similarly, we can also calculate a MPM of the class $C_{k}$ using the SAM, i.e.,

$$
\pi_{k}^{A}(\mathbf{x})=\frac{\exp \left(\frac{-S A M\left(\mathbf{f}_{\lambda}(\mathbf{x}), \mu_{k}\right)}{\sigma_{M P M}}\right)}{\sum_{\mathbf{y} \in E} \exp \left(\frac{-S A M\left(\mathbf{f}_{\lambda}(\mathbf{x}), \mu_{k}\right)}{\sigma_{M P M}}\right)} .
$$

A comparison of MPM using Euclidean distance vs. SAM, for four spectral classes of "Indian Pines", is given in Fig. 3. On the basis of these examples, we conclude that for our purpose both approaches $\pi_{k}^{M}(\mathbf{x})$ and $\pi_{k}^{A}(\mathbf{x})$ lead to quite similar results and hence, for the experiments of this paper we will consider only $\pi_{k}^{M}(\mathbf{x})$, denoted from now on by $\pi_{k}(\mathbf{x})$.

\section{REGIONALIZED RANDOM GERMS SIMULATIONS FROM THE MPMS}

We consider in this section the theoretical distribution which follows the regionalized random points as well as the algorithm used to simulate a realization of $N$ random germs associated to each MPM.

\subsection{Regionalized Poisson points}

A rather natural way to introduce uniform random germs is to generate realizations of a Poisson point process with a constant intensity $\theta$, namely average number of points per unit area. It is well known that the random number of points $N(D)$ falling in a domain $D$, which is considered a bounded Borel set, with area $|D|$, follows

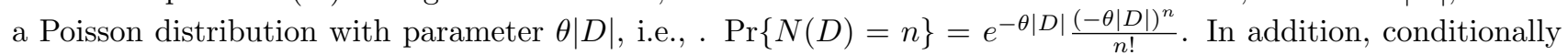
to the fact that $N(D)=n$, the $n$ points are independently and uniformly distributed over $D$, and the average number of points in $D$ is $\theta|D|$ (i.e., the mean and variance of a Poisson distribution is its parameter).

More generally, we can suppose that the density $\theta$ is not constant; but considered as measurable function, defined in $\mathbb{R}^{d}$, with positive values. For simplicity, let us write

$$
\theta(D)=\int \theta(\mathbf{x}) d \mathbf{x}
$$

It is also known ${ }^{6,7}$ that the number of points falling in a borel set $B$ according to a regionalized density function $\theta$ follows a Poisson distribution of parameter $\theta(D)$, i.e.,

$$
\operatorname{Pr}\{N(D)=n\}=e^{-\theta(D)} \frac{(-\theta(D))^{n}}{n !} .
$$

In such a case, if $N(D)=n$, the $n$ are independently distributed over $D$ with the probability density function $\widehat{\theta}(\mathbf{x})=\theta(\mathbf{x}) / \theta(D)$. 


\subsection{Algorithm for simulation of germs for each spectral class}

We have proposed above that each spectral class $C_{k}$ of the hyperspectral image is modelled according to a MPM $\pi_{k}(\mathbf{x})$; we notice that in this case the domain $D$ corresponds to the discrete support space of the image. In what follows, we are interested in generating a non-uniform distribution of germs, with a regionalized probability density function associated to class $k$ such that $\widehat{\theta}(\mathbf{x}) \equiv \pi_{k}(\mathbf{x})$.

In practice, in order to simulate a realization of $N$ independent random germs distributed over the image with the pdf $\pi_{k}(\mathbf{x})$ we propose to use an inverse transform sampling method. More precisely, the algorithm to generate $N$ randoms germs in an image $m(\mathbf{x})$ according to density $\widehat{\theta}(\mathbf{x})$ is as follows:

1. Initialization: $m\left(\mathbf{x}_{i}\right)=0 \forall \mathbf{x}_{i} \in E ; P=\operatorname{Card}(E)$

2. Compute cumulative distribution function: $c d f\left(\mathbf{x}_{i}\right)=\frac{\sum_{k \leq i} \widehat{\theta}\left(\mathbf{x}_{k}\right)}{\sum_{k=1}^{P} \widehat{\theta}\left(\mathbf{x}_{k}\right)}$

3. for $j=1$ to $N$

4. $r_{j} \sim \mathcal{U}(1, P)$

5. Find the value $s_{j}$ such that $r_{j} \leq c d f\left(\mathbf{x}_{s_{j}}\right)$.

6. $m\left(\mathbf{x}_{s_{j}}\right)=1$

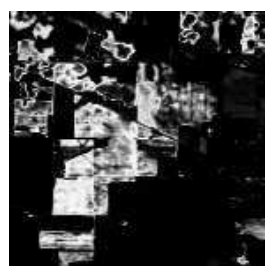

(a1)

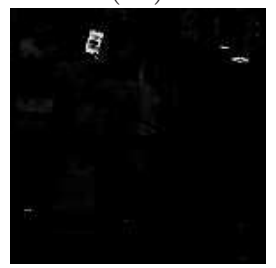

$(\mathrm{a} 2)$

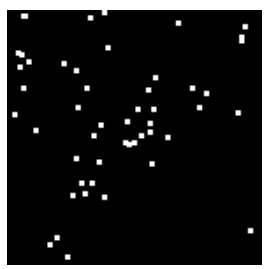

(b1)

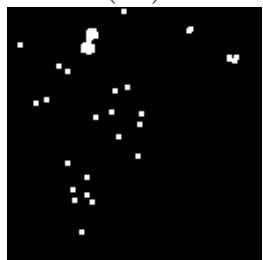

(b2)

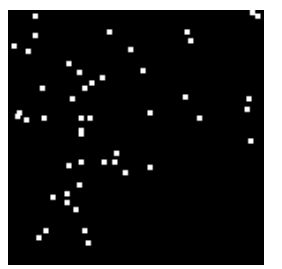

(c1)

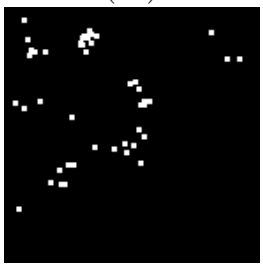

(c2)

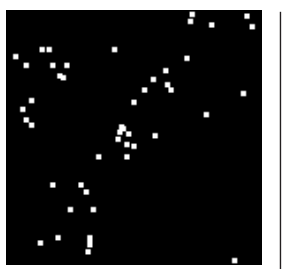

(d1)

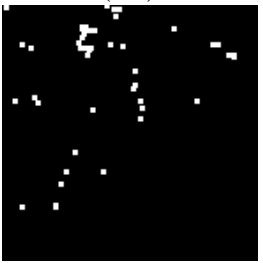

$(\mathrm{d} 2)$

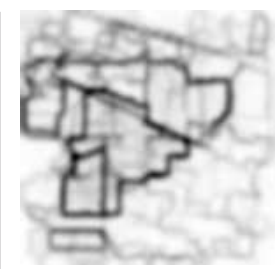

$(\mathrm{e} 1)$

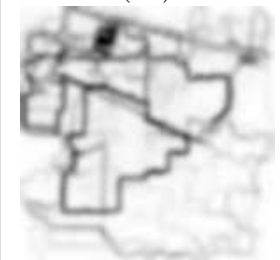

$(\mathrm{e} 2)$

Figure 4. MonteCarlo estimation of pdf of contours using SW for two spectral classes of "Indian Pines" (first row corresponds to class 2 and second row to class 16): (a) MPM of the class $\pi_{k}(\mathbf{x})$; (b), (c) and (d) three realizations of 50 regionalized random points $m r k_{i}^{\pi_{k}}(\mathbf{x})$ (the points have been dilated for a better visualization); (e) semi-supervised pdf of contours $p d f^{C_{k}}(\mathbf{x})$ obtained from 50 realizations (in negative, the dark values correspond to high probabilities).

\section{SEMI-SUPERVISED PROBABILITY DENSITY OF CONTOURS USING STOCHASTIC WATERSHED}

We have now all the ingredients for the semi-supervised SW. We start by considering that a MPM image is constructed for each spectral class of the hyperspectral image, i.e., $\left\{\mathbf{f}_{\lambda}(\mathbf{x}), C_{k}\right\} \mapsto \pi_{k}(\mathbf{x})$. As mentioned above, $\pi_{k}(\mathbf{x})$ is obtained in all the experiments using the Euclidean distance, where the mean spectrum of the class is computed from only 10 random training spectra of the class. Then, the MPM of each class is used as a regionalized density function to generate $M$ realisations of $N$ random points, i.e., $\pi_{k}(\mathbf{x}) \mapsto\left\{m r k_{i}^{\pi_{k}}(\mathbf{x})\right\}_{i=1}^{M}$. These images of random markers are employed to calculate the $M$ realisations of watershed lines needed in the MonteCarlo procedure for estimation of the probability density of contours of spectral band $\lambda_{j}$ with respect to the class $C_{k}$, i.e.,

$$
p d f_{\lambda_{j}}^{C_{k}}(\mathbf{x})=\frac{1}{M} \sum_{i=1}^{M} W S\left(\varrho\left(f_{\lambda_{j}}\right), m r k_{i}^{\pi_{k}}\right)(\mathbf{x}) * K\left(\mathbf{x} ; \sigma_{\text {spatial }}\right)
$$


Finally, as in the standard case, the pdf of the hyperspectral image w.r.t. the class $C_{k}$ is obtained as the kernelized sum of the marginal pdf's of the spectral bands, i.e.,

$$
\left\{\mathbf{f}_{\lambda}(\mathbf{x}), C_{k}\right\} \mapsto p d f^{C_{k}}(\mathbf{x})=\frac{1}{L} \sum_{j=1}^{L} p d f_{\lambda_{j}}^{C_{k}}(\mathbf{x}) * K\left(\lambda ; \sigma_{\text {spectral }}\right) .
$$

Examples of this MonteCarlo estimation of pdf of contours using SW, for two spectral classes of "Indian Pines", are provided in Fig. 4; in particular, 3 realizations of 50 regionalized random points for each MPM are depicted. The pdf's are estimated with 50 realizations of SW. We can observe that the contours of high probability from the pdf of each class correspond mainly to regions of the image associated to the spectral class according, of course, to the spectral discrimination specificity provided by the MPM. The class 16 ("Stone-steel towers") of "Indian Pines" is a quite particular case which will be discussed in next section.

Once the pdf of the $K$ classes has been computed, it is obvious that we can define a global pdf of the hyperspectral image as an average of the pdf of the different classes, i.e.,

$$
p d f^{C}(\mathbf{x})=\frac{1}{K} \sum_{k=1}^{K} p d f^{C_{k}}(\mathbf{x})
$$

We must remark the this regionalized multi-class $p d f^{C}(\mathbf{x})$ is different from that obtained using uniform points $p d f(\mathbf{x})$, as discussed in Section 2. A comparison of both global pdf's with respect to those of various spectral classes of "Indian Pines" are given in Fig. 5. From the examples we can state that, using the semi-supervised MonteCarlo framework of stochastic watershed, the image zones associated to the particular spectral class, which drove the simulation procedure, are enhanced with respect to the other zones. That involves that the contours of higher probability correspond to zones associated to the particular spectral class. However, as we can observe from the examples, these zones are not segmented as a single "region"; on the contrary, they are "segmented" in their various structural regions. Or in other words, zones which appear as a single connected component in the spectral ground truth are segmented in various spatial connected components.
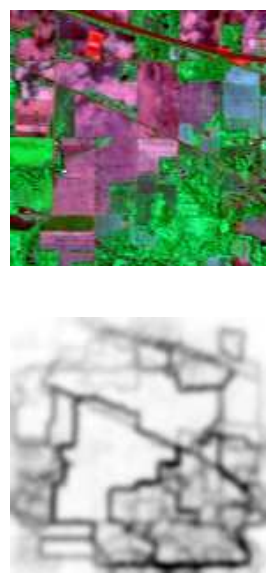

(d)

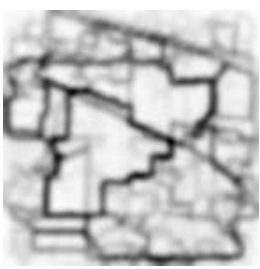

(a)

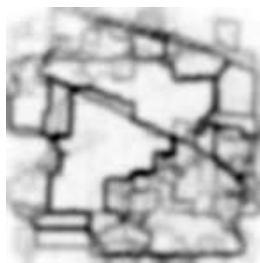

(e)

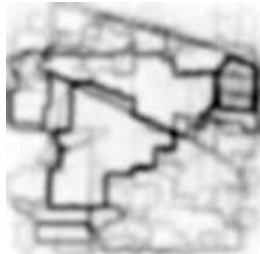

(f)

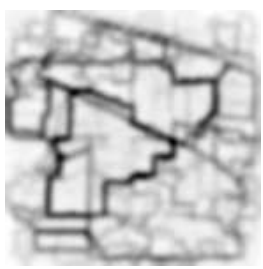

(b)

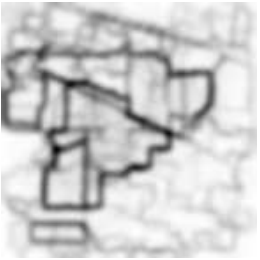

$(\mathrm{g})$

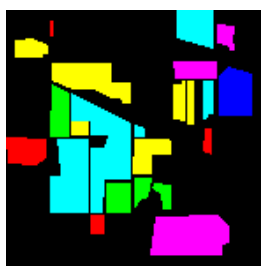

(c)

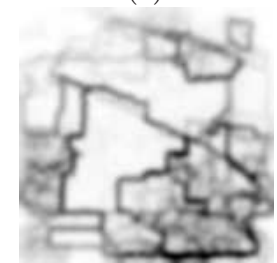

(h)

Figure 5. MonteCarlo estimation of pdf's of contours using SW for "Indian Pines": (a) uniform unsupervised pdf of contours $p d f(\mathbf{x})$; (b) semi-supervised multi-class pdf of contours $p d f^{C}(\mathbf{x})$; (c) ground truth of some classes (in Yellow class 2 "Corn-notill", in Red class 5 "grass/pasture", in Green class 6 "grass/trees", in Blue class 8 "hay-windrowed", in Cyan class 11 "soybeans-min", in Magenta class 14 "woods"). (d) semi-supervised pdf of contours of class 5 pdf ${ }^{C_{5}}(\mathbf{x}),(\mathrm{e})$ semisupervised pdf of contours of class $6 p d f^{C_{6}}(\mathbf{x})$, (f) semi-supervised pdf of contours of class $8 p d f^{C_{8}}(\mathbf{x})$, (g) semi-supervised pdf of contours of class $11 p d f^{C_{11}}(\mathbf{x})$, (h) semi-supervised pdf of contours of class $14 p d f^{C_{14}}(\mathbf{x})$. 


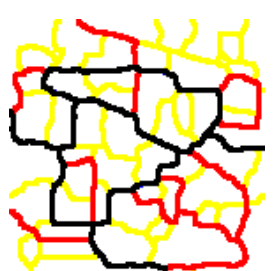

(a1)

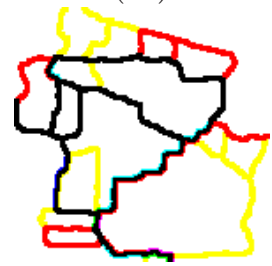

(b1)

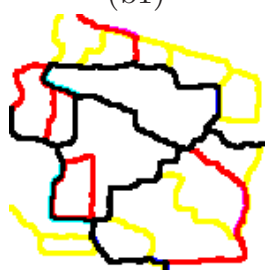

(c1)

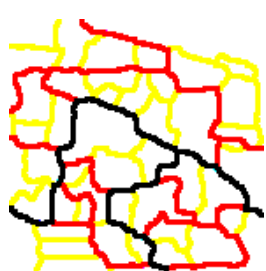

(a2)

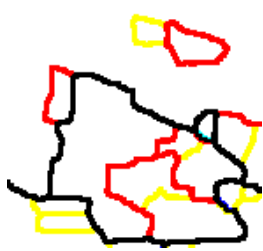

(b2)

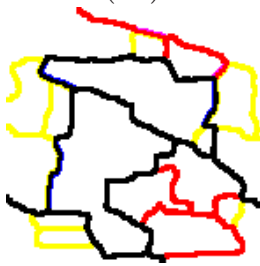

(c2)

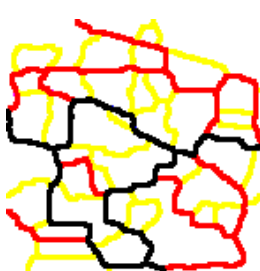

(a3)

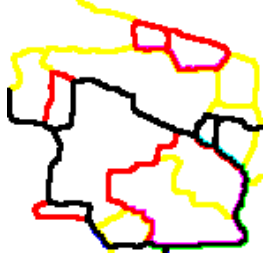

(b3)

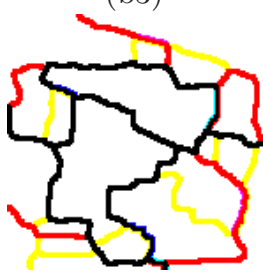

(c3)

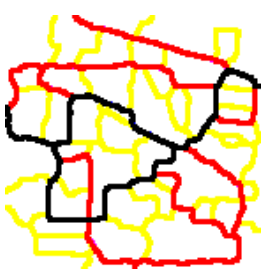

(a4)

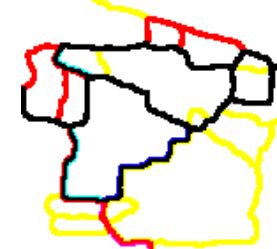

(b4)

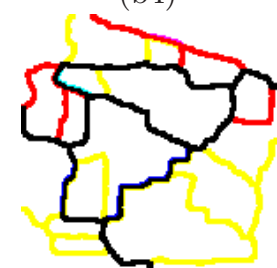

(c4)

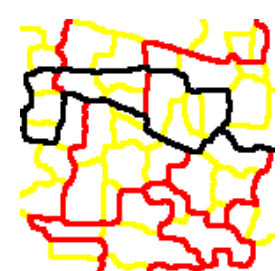

(a5)

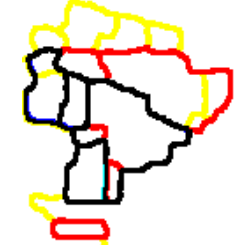

(b5)

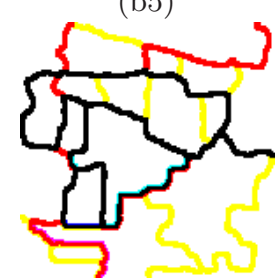

(c5)

Figure 6. Hierarchical segmentation of semi-supervised pdf's of contours for "Indian Pines" (first column corresponds to multi-class $p d f^{C}(\mathbf{x})$, second column to spectral class $5 p d f^{C_{5}}(\mathbf{x})$, third column to spectral class $6 p d f^{C_{6}}(\mathbf{x})$, fourth column to spectral class $8 p d f^{C_{8}}(\mathbf{x})$ and fifth column to spectral class $11 p d f^{C_{11}}(\mathbf{x})$ ): (a) three levels of waterfalls algorithm (first in yellow, second in red and third in black); (b) three levels of dynamics-based segmentation in 5 regions (in yellow), 10 regions (in red) and 20 regions (in black); (c) three levels of volumic-based segmentation in 5 regions (in yellow), 10 regions (in red) and 20 regions (in black). The corresponding pdf's are given in Fig. 5.

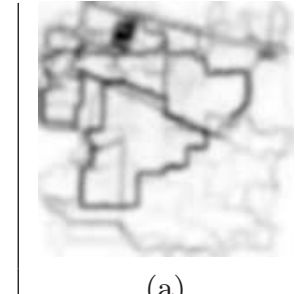

(a)

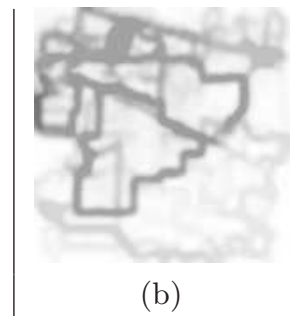

(b)

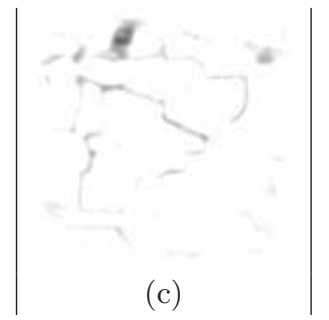

(d)

Figure 7. Example of target region detection from semi-supervised pdf: (a) Initial image is the complemented pdf $t_{0}(\mathbf{x})=$ $-p d f^{C_{16}}(\mathbf{x})$, (b) close-holes operator $t_{1}(\mathbf{x})=$ Cl-Holes $\left(t_{0}(\mathbf{x})\right)$, (c) negative of residue $t_{2}(\mathbf{x})=-\left|t_{0}(\mathbf{x})-t_{1}(\mathbf{x})\right|,(\mathrm{d})$ closing $t_{3}(\mathbf{x})=\varphi_{B}\left(t_{2}(\mathbf{x})\right)$ where $B$ is a disk of 8 pixels.

\section{PDFS POST-PROCESSING AND HIERARCHICAL SEGMENTATION}

The $p d f^{C_{k}}(\mathbf{x})$ could be directly thresholded in order to obtain the most prominent contours, however the results are only pieces of contours (not enclosing regions). In addition, we have studied the histograms for several examples and there is not an optimal threshold to separate the classes of contours.

Let us start here by considering again the example of the pdf of class 16 . The ground truth corresponds to a well defined spectral region which involves also a well defined spatial region of high probability in the pdf. Such a case, which can be considered as a problem target region detection, may be solved using standard morphological operators. ${ }^{14}$ In particular, the simple algorithm given in Fig. 7, based on classical operators like the close-holes or closing using a disk as structuring element, illustrates how a region of a particular size/shape and a minimal value of probability detection can be extracted. 
"Indian Pines"

\begin{tabular}{|c|c|c|c|c|c|c|c|c|}
\hline & Multi-Class & Class 2 & Class 5 & Class 6 & Class 8 & Class 10 & Class 11 & Class 16 \\
\hline$\mu(\mathrm{Pr})$ & 0.30 & 0.39 & 0.27 & 0.48 & 0.70 & 0.33 & 0.43 & 0.43 \\
Sensit & 83.76 & 82.44 & 84.16 & 91.05 & 21.27 & 95.81 & 59.01 & 84.30 \\
Specif & 51.42 & 51.52 & 51.88 & 51.82 & 51.93 & 51.75 & 51.29 & 51.96 \\
\hline \\
\begin{tabular}{|c|c|c|c|c|c|c|c|}
\hline \\
\hline
\end{tabular} & Multi-Class & Class 1 & Class 2 & Class 3 & Class 4 & Class 7 & Class 8 & Class 9 \\
\hline Sensit. & 0.17 & 0.31 & 0.22 & 0.17 & 0.28 & 0.36 & 0.31 & 0.49 \\
Specif. & 90.66 & 32.82 & 15.00 & 3.56 & 30.25 & 55.39 & 88.61 & 49.13 \\
& 52.93 & 81.81 & 80.97 & 81.71 & 82.96 & 52.27 & 52.20 & 51.90 \\
\hline
\end{tabular}

Table 1. Quantitative assessment of segmentation results for "Indian Pines" (segmentation of pdf's in 100 dynamics-based regions) and "Pavia" (segmentation of pdf's in 20 dynamics-based regions).

More generally, an alternative technique to segment automatically the pdf in significant closed regions is to apply a morphological hierarchical algorithm. Several watershed-based hierarchical approaches allow addressing imaging problems where the markers cannot be easily defined. Mainly, two hierarchical techniques can be distinguished:

i) Non-parametric waterfalls algorithm; ${ }^{4}$

ii) Hierarchies based on extinction values, which allows to select the minima used in the watershed according to morphological criteria (dynamics, surface area and volume). ${ }^{10,17}$

These methods of hierarchical segmentation have been used to segment the semi-supervised pdf's of contours of "Indian Pines". In Fig. 6 is given a systematic comparison for the pdf's of four spectral classes together with the multi-class pdf. More precisely, it is shown the three first levels of waterfalls algorithm (first in yellow, second in red and third in black); three levels of dynamics-based segmentation in 5 regions (in yellow), 10 regions (in red) and 20 regions (in black) and three levels of volumic-based segmentation in 5 regions (in yellow), 10 regions (in red) and 20 regions (in black). In the three cases the obtained segmentations are coherent with the expected results according to the semi-supervised pdf's. We observe also that the hierarchical segmentation for the multi-class pdf gives the most important image regions independently of the spectral behaviour. It seems in any case that, for the purpose of class-driven segmentation, the hierarchies based on the dynamics are particularly interesting. Indeed, the same algorithms of regionalized stochastic watershed have been also applied to the image "Pavia". In Fig. 8 are depicted the results for a crop of the image. The comparison includes the multi-class pdf and seven of the semi-supervised spectral pdf's (the image has nine spectral classes) as well as their hierarchical segmentations using dynamics criterion in 25 regions (in yellow), 50 regions (in red) and 100 regions (in black). We observe the visual quality of the semi-supervised pdf's and subsequent segmentations are relatively good, taking into account the structural complexity of the image.

By selecting a particular level of the dynamics-based hierarchy, which involves selecting a priori the number of regions, a more quantitative assessment of the segmentation results can be achieved. More precisely, using the ground truth segmentations of each class, we have compared quantitatively the performances by computing three parameters: i) the average probability of $p d f^{C_{k}}$ on the ground truth contours, denoted by $\mu(\operatorname{Pr})$, ii) the sensitivity percentage $S e n s i t=100 T P /(T P+F N)$, iii) the specificity percentage Specif $=100 T N /(T N+F P)$; (where $T P, T N, F P, F N$ are defined in terms of pixels of the contours). The values for a both images are given in the Table 1. As we can observe, the quantitative results for "Indian Pines" are better than for "Pavia": the reference contours of spectral regions of "Pavia" are often associated to narrow regions and their correspondence with the obtained contours is not always perfectly located. In addition, we notice again that these results correspond to a segmentation in a fixed number of regions. More evolved techniques for an accuracy assessment of the segmentation, as recently proposed in, ${ }^{13}$ can be used for a most systematic evaluation of results and in order to optimize the parameters for particular applications.

\section{CONCLUSION AND PERSPECTIVES}

We have introduced in this paper a novel probabilistic framework for semi-supervised image segmentation in high spatial resolution hyperspectral images using stochastic watershed, and without using classification tech- 
niques. The required information is a training set of representative spectra of each spectral class and produces a probability density function of contours for each class, plus a global multi-class pdf.

We can conclude the empirical study by saying that our approach of stochastic segmentation performs relatively well, except for image regions with very irregular contours or regions associated to spectral classes which are not well sampled during the regionalized simulation procedure. In fact, the main limitation of our approach is given by the simplicity of modelling the membership probability map, which involves an unspecific regionalized simulation of germs. If this step is improved, using advanced machine learning techniques the quality of the final pdf, and subsequent segmentation, would be also improved. In addition, work remains to be done in the integration of the semi-supervised spatial information, associated to the probability of a pixel to belong to a contour of a particular spectral class, with the spectral information of the pixel used for classification. A promising direction is to use graph-based semi-supervised image segmentation techniques integrating spatial-spectral kernels. ${ }^{18}$

Acknowledgment. The authors would like to thank the IAPR-TC7 for providing the data, and Prof. P. Gamba and Prof. F. Dell'Acqua of the University of Pavia, Italy, for providing reference data.

\section{REFERENCES}

[1] J. Angulo, D. Jeulin. Stochastic watershed segmentation. In Proc. Int. Symp. Mathematical Morphology ISMM'07, Rio, Brazil, INPE, Eds. Banon, G. et al., 265-276, 2007.

[2] J. Angulo, S. Velasco-Forero, J. Chanussot. Multiscale stochastic watershed for unsupervised hyperspectral image segmentation. In Proc. of IEEE IGARSS'2009, Vol. III, 93-96, Cape Town, South Africa, July 2009.

[3] S. Beucher, F. Meyer. The Morphological Approach to Segmentation: The Watershed Transformation. In Mathematical Morphology in Image Processing, Marcel Dekker, Eds. E. Dougherty, 1992, 433-481, 1992.

[4] S. Beucher. Watershed, hierarchical segmentation and waterfall algorithm. In Mathematical Morphology and its Applications to Image and Signal Processing, Proc. ISMM'94 Kluwer, 69-76, 1994.

[5] O. Chapelle, B. Schölkopf and A. Zien. Semi-Supervised Learning. MIT Press, Cambridge, MA, 2006.

[6] C. Lantuéjoul. Geostatistical Simulation: Models and Algorithms, Springer-Verlag, Heidelberg, Berlin, 2002.

[7] G. Matheron. Random sets and integral geometry, Wiley, New York, 1975.

[8] D.G. Manolakis, V.K. Ingle, S.M. Kogon. Statistical and Adpative Signal Processing: Spectral Estimation, Signal Modelling, Adaptive Filtering and Array Processing, McGraw-Hill, Boston, 2000.

[9] D.G. Manolakis, C. Siracusa, G. Shaw. Hyperspectral Subpixel Target Detection Using the Linear Mixing Model. IEEE Trans. on Geoscience and Remote Sensing, Vol. 39, No. 7, 2001.

[10] F. Meyer. An Overview of Morphological Segmentation. International Journal of Pattern Recognition and Artificial Intelligence Vol. 15, No. 7, 1089-1118, 2001.

[11] G. Noyel, J. Angulo, D. Jeulin. Random Germs and Stochastic Watershed for Unsupervised Multispectral Image Segmentation. In Proc. KES 2007/ WIRN 2007, Part III, LNAI 4694, Springer, 17-24, 2007.

[12] G. Noyel, J. Angulo, D. Jeulin. Classification-driven stochastic watershed. Application to multispectral segmentation. In Proc. of the ISET's Fourth European Conference on Color on Graphics, Imaging and Vision (CGIV'2008), Terrasa-Barcelona, Spain, 471-476, 2008.

[13] C. Persello, L. Bruzzone. A Novel Protocol for Accuracy Assessment in Classification of Very High Resolution Images. IEEE Trans. on Geoscience and Remote Sensing, Vol. 48, No. 3, 1232-1244, 2010.

[14] P. Soille. Morphological image analysis, Springer-Verlag, Berlin Heidelberg, 1999.

[15] Y. Tarabalka, J. Chanussot, J.-A. Benediktsson, J. Angulo, M. Fauvel. Segmentation and classification of hyperspectral data using watershed. In Proc. of IEEE IGARSS'08, Boston-Massachusetts, USA, July 2008.

[16] Y. Tarabalka, J. Chanussot, J.-A. Benediktsson. Segmentation and classification of hyperspectral images using watershed transformation. Pattern Recognition, 2010.

[17] C. Vachier, F. Meyer. Extinction value: a new measurement of persistence. IEEE Workshop on Nonlinear Signal and Image Processing, pp. 254-257, 1995.

[18] S. Velasco-Forero, V. Manian. Improving Hyperspectral Image Classification Using Spatial Preprocessing. IEEE Geoscience and Remote Sensing Letters, 6(2): 297-301, 2009.

[19] R.H. Yuhas, A.F.H. Goetz, J.W. Boardman. Discrimination among semiarid landscape endmembers using the spectral angle mapper (SAM) algorithm. In Summaries of the Third Annual JPL Airborne Geoscience Workshop, JPL Publication 92-14, Vol. 1, 147-149, 1992. 

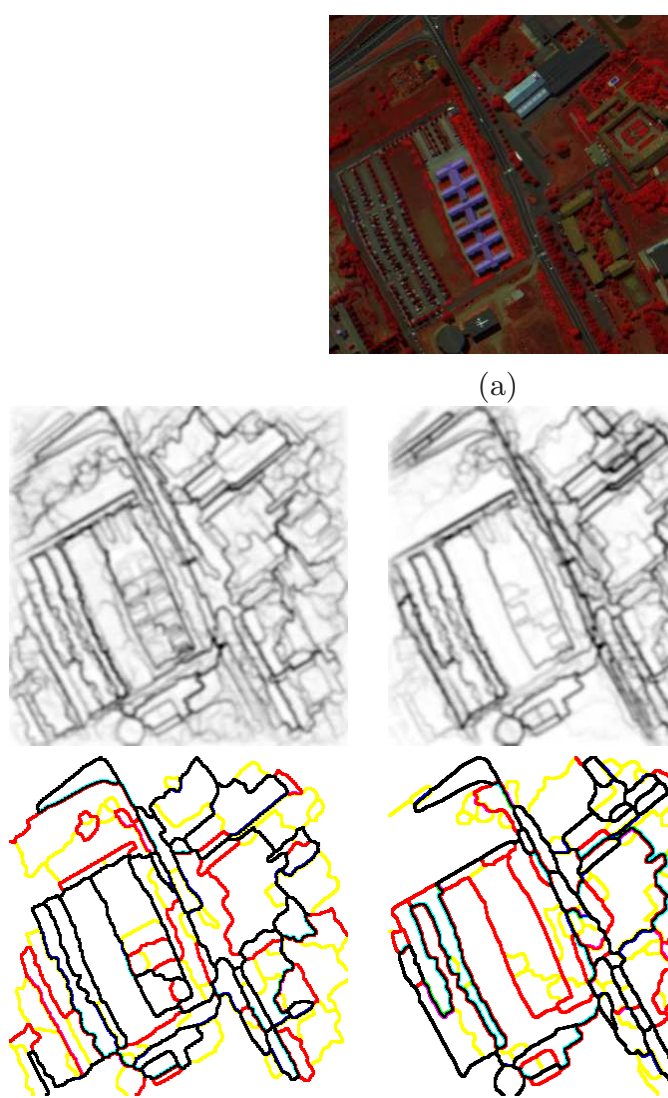

(c)
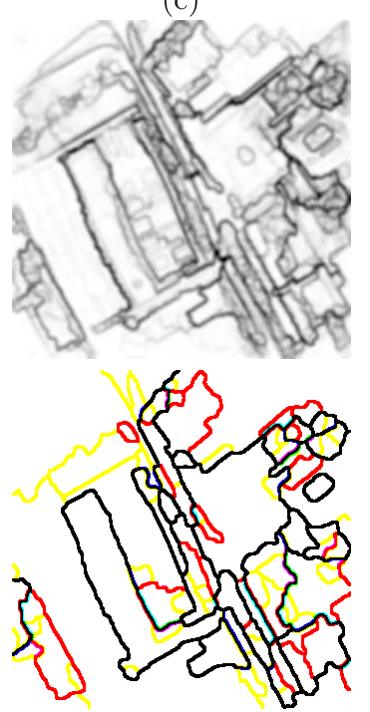

(g) (a)
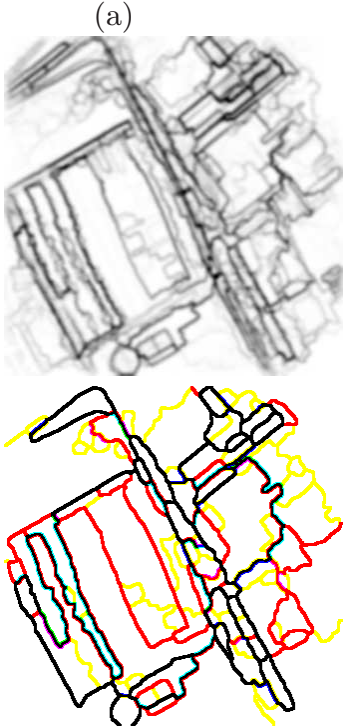

(d)
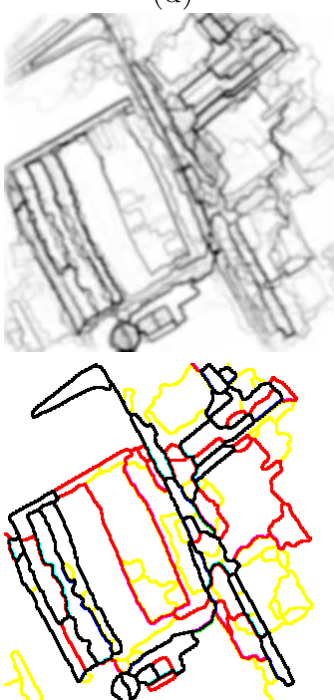

(h)
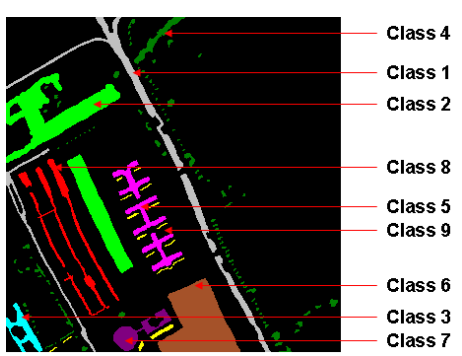

(b)
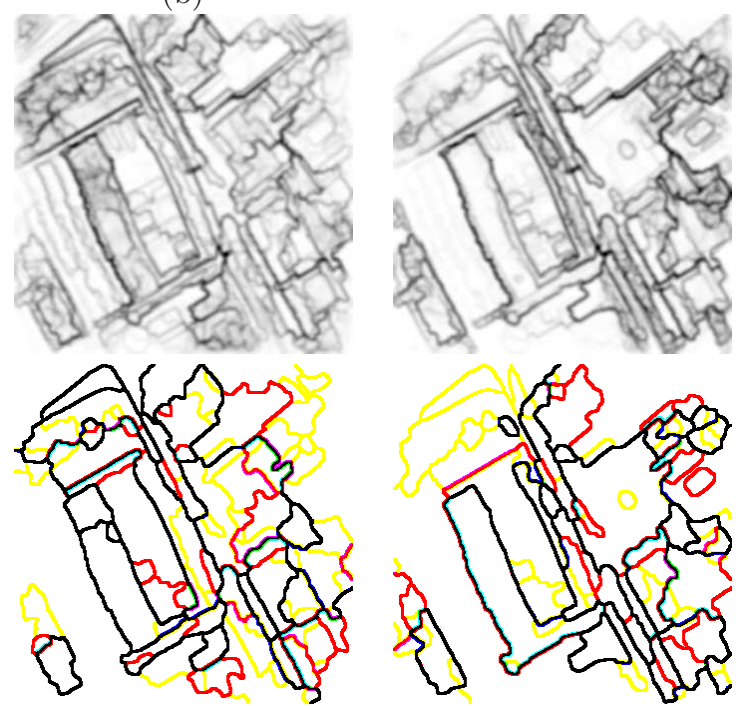

(e)
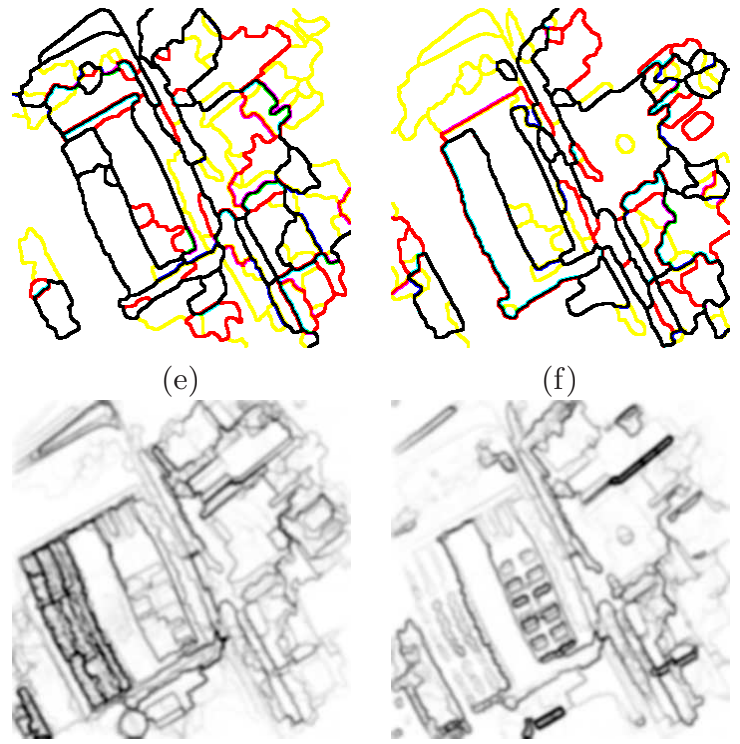

(f)
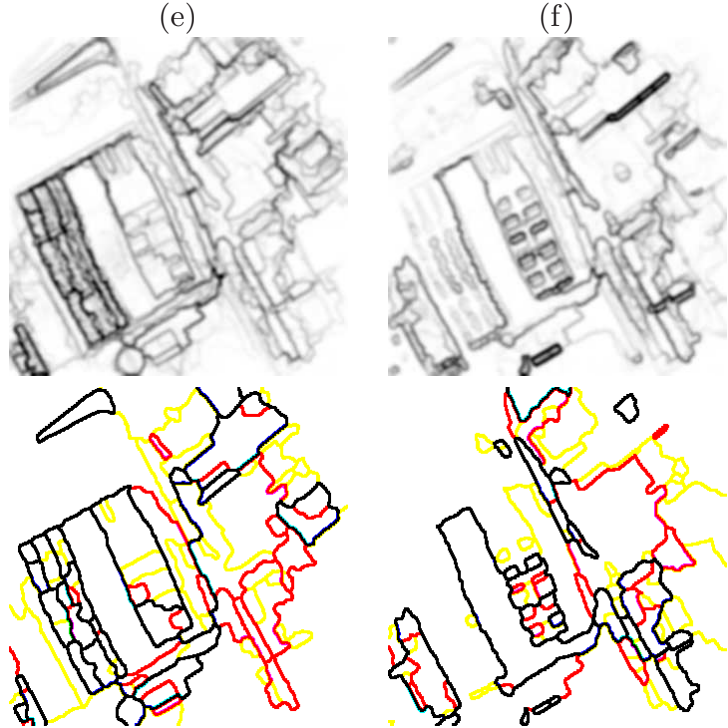

(i)

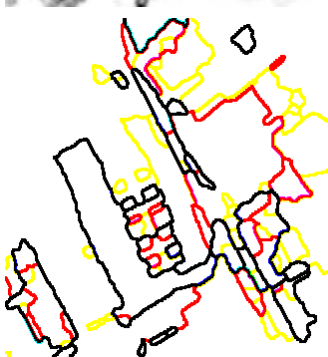

(j)

Figure 8. Semi-supervised spectral-driven pdf's of contours for a part of the hyperspectral imge "Pavia" and their hierarchical segmentations using dynamics criterion in 25 regions (in yellow), 50 regions (in red) and 100 regions (in black): (a) original image from significant spectral bands, (b) ground truth for the nine spectral classes, (c) multi-class $p d f^{C}(\mathbf{x})$, (d) spectral class $1 p d f^{C_{1}}(\mathbf{x}),(\mathrm{e})$ spectral class $2 p d f^{C_{2}}(\mathbf{x}),(\mathrm{f})$ spectral class $3 p d f^{C_{3}}(\mathbf{x})$, (g) spectral class $4 p d f^{C_{4}}(\mathbf{x}),(\mathrm{h})$ spectral class $7 p d f^{C_{7}}(\mathbf{x})$, (i) spectral class $8 p d f^{C_{8}}(\mathbf{x}),(\mathbf{j})$ spectral class $9 p d f^{C_{9}}(\mathbf{x})$. 